\title{
BANCO DE SEMENTES DE UMA FLORESTA SECUNDÁRIA AMAZÔNICA DOMINADA POR Vismia
}

\author{
Luciana M. MONACO ${ }^{1}$, Rita C. G. MESQUITA ${ }^{1,2}$, G. Bruce WILLIAMSON ${ }^{3}$
}

RESUMO: A composição florística de uma floresta secundária dominada por Vismia na Amazônia central foi estudada e comparada de acordo com sua composiçāo ao banco de sementes no solo. A área apresentou um total de 20 espécies arbóreas, sendo Vismia o gênero dominante em indíce de valor de importância, densidade relativa e área basal. $\mathrm{O}$ banco de sementes coletado na área apresentou germinaçāo de apenas 17 espécies, todas pioneiras, entre as quais metade foi de herbáceas e a outra metade de lenhosas. A composição do banco de sementes foi em geral característica de estágios sucessionais, com forte influência do histórico de pastagem, presença de espécies pioneiras e um elevado número de herbáceas e gramíneas. Os gêneros Goupia e Laetia estiveram ausentes no banco mesmo com indivíduos adultos representados no dossel. Os três gêneros com maior representaçāo no banco de sementes em relação ao dossel foram Cecropia, Bellucia e Trema, os quais provavelmente germinaram de sementes chegadas depois do desenvolvimento do dossel de Vismia. As parcelas que tiveram o dossel removido refletiram muito bem a composiçāo da vegetaçāo estabelecida na área, onde sementes de espécies da floresta mais madura não germinaram. Desta forma, o retorno à floresta primária deverá ser um processo lento e dependente de fontes de sementes próximas, além da presença de dispersores capazes de contribuir com a chuva de sementes.

PALAVRAS-CHAVE: Banco de sementes, pioneiras, Amazônia, mata secundária, sucessão, regeneração, Vismia

\section{THE SEED BANK OF A SECONDARY AMAZONIAN FOREST DOMINATED BY Vismia}

ABSTRACT : The floristic compostion of a secondary forest dominated by Vismia in central Amazonia was studied and compared to the composition of the seed bank in the soil. The area contained 20 tree species, with Vismia being the dominant genus in importance value, relative density, and basal area. The seed bank collected from the area exhibited 17 species that germinated, all pioneers, of which half were herbaceous and half were woody. In general, the composition of the seed bank was characteristic of successional stages with a strong influence from the abandoned pasture, the presence of pioneer species, and a large number of herbs and grasses. Two genera, Goupia and Laetia, were absent from the bank, although adult individuals were in the canopy. The three genera over-represented in the seed bank,

\footnotetext{
${ }^{1}$ Departamento de Ekologia, Instituto Nacional de Fesquisas da Amazônia, CP 478, 69011-970 Menaus, Amazcras, Basil

${ }^{2}$ Projeto Dinêmica Biológica dos Fragnentos Florestais, INEA, (P 478, 69011-970 Menaus, Amazonas, Brasil

${ }^{3}$ Department of Biological Scienes, Loisiana State Lriversity, Baton Rage, Iouisiam 70803, FIA
} 
relative to the canopy, were Cecropia, Bellucia and Trema, whose seeds probably arrived after the development of the canopy of Vismia. In field plots where the canopy was removed, germination mirrored the composition of the established vegetation in the area without the presence of new species exclusive to the seed bank. Thus, the return to primary forest will be delayed and depend on the proximity of seed sources as well as the presence of dispersers to contribute a diverse seed rain.

KEYWORDS: Seed bank, pioneers, Amazonia, secondary forest, regeneration, succession, Vismia

\section{INTRODUÇÃO}

A regeneração de áreas desmatadas provêm de quatro fontes básicas: o rebroto de partes da planta restante, a regeneração avançada (banco de plântulas), o banco de sementes e a chuva de sementes (dispersão). As sementes enterradas no solo e as que estão na superfície compõem o banco de sementes (Garwood, 1989), que pode ser definido como o "pool" de sementes viáveis presentes no solo (Vieira, 1996).

A composição do banco de sementes não reflete a composição florística da mata primária (Cheke et al., 1979; Thompson, 1979; Leal, 1992; Vieira, 1996), pois as espécies pioneiras geralmente dominam o banco de sementes em áreas tropicais. A maioria das espécies pioneiras produzem sementes que permanecem longos períodos no solo por apresentar algum tipo de dormência.

Em geral, espécies pioneiras acumulam mais sementes no banco do que espécies tolerantes à sombra, muito embora existam sementes de mata primária que persistem por longos períodos nos solos. As espécies pioneiras têm efetivos mecanismos de dispersāo à longa distância e uma produçâo de sementes precoce e em grande escala, o que incrementa a entrada de sementes no solo via chuva de sementes (Uh1 \& Clark, 1983; Rodrigues et al., 1990).

De acordo com Thompson (1992), a permanência no solo pode ser transicional (menos de 1 ano no solo), pouco persistente (entre 1-5 anos) ou muito persistente (no mínimo 5 anos). A dormência no solo pode ser afetada por requerimentos de germinação, como a incidência luminosa e proporção de luz infravermelha e vermelha longo (Vazquez-Yanes, 1980; Holthuijzen \& Boerboom, 1982).

A proporçāo de sementes viáveis nos solos diminui de acordo com a profundidade. Existem mais sementes viáveis nas camadas superficiais do solo (até $5 \mathrm{~cm}$ ) do que nas camadas mais profundas, pois as sementes mais superficiais germinam mais facilmente que as enterradas abaixo de $10 \mathrm{~cm}$ (Garwood, 1989; Leal, 1992; Dalling et al., 1994; Vieira, 1996). Muito embora existam registros de um grande número de sementes viáveis a profundidades diversas.

A composição e a densidade de sementes oriundas da chuva de sementes variam em função da proximidade da mata e de zonas agropecuárias. Áreas de vegetação secundária próximas a áreas de cultivo e pastagens têm maior número de ervas e cipós do que clareiras naturais. À medida que a vegetação secundária se aproxima da mata, a composição específica 
das sementes muda, diminuindo os taxa herbáceos e aumentando os arbóreos (Gorchov et al., 1993).

$O$ estudo do banco de sementes pode dar informações sobre a densidade de sementes, composição florística e dar uma indicaçāo do potencial regenerativo das sementes estocadas nos solos (WilliamsLinera, 1993). Já foi mostrado que o recrutamento vegetal em floresta secundária na Amazônia central dependerá do gênero que a coloniza, Vismia ou Cecropia. Por exemplo, a riqueza de espécies sob o dossel de Cecropia é duas vezes maior do que o de Vismia (Williamson et al., 1998; Mesquita et al., 2001). O objetivo deste trabalho foi descrever o banco de sementes de uma floresta secundária dominada por Vismia (Clusiaceae) oriunda de pastagem abandonada. Assim, os objetivos deste estudo foram: (1) comparar o número de indivíduos e espécies presentes no banco de sementes com a vegetação presente; e (2) comparar os resultados de uma coleta instantânea, mantida em casa de vegetação, com parcelas monitoradas diretamente no campo.

\section{MATERIAL E MÉTODOS}

A área estudada foi uma vegetação secundária, dominada por Vismia, à $65 \mathrm{~km}$ ao norte da cidade de Manaus, no estado do Amazonas, Brasil. A área faz parte da Fazenda Esteio (224' 48” S, 5952' 21” W), localizada à $23 \mathrm{~km}$ da estrada vicinal ZF-3, pertencente ao Distrito Agropecuário da Suframa, e faz parte do Projeto Dinâmica Biológica dos Fragmentos Florestais (PDBFFINPA) (Lovejoy \& Bierregaard, 1990). As parcelas estudadas se situam $500-600 \mathrm{~m}$ ao norte da Reserva 1202 de 10 ha do PDBFF.
A área foi desmatada em 1980, queimada e comvertido em pastagem. Foi utilizada moderadamente como pastagem por alguns anos, sofrendo a última queima em 1989, seguida de abandono. Portanto, a vegetação no início do experimento em 1996 tinha aproximadamente 7 anos de idade e seu dossel era dominado por espécies de Vismia.

Os solos da região são argilosos, classificados como latossolo amarelo e podzólico vermelho-amarelo. O relevo é predominantemente plano com algumas áreas íngremes próximas a igarapés. A precipitação anual pode variar entre $1900-2500 \mathrm{~mm}$ com uma estaçāo seca de junho a outubro (Rankin-deMerona et al., 1992). No ano deste estudo, 1997, em decorrência do fenômeno climático El Niño, a estaçāo seca foi mais pronunciada que o normal, porém a estação chuvosa anterior foi bastante intensa, produzindo um balanço de precipitação anual total de $2024 \mathrm{~mm}$.

\section{Composição da floresta secundária}

Foram estabelecidas aleatoriamente 8 parcelas de $15 \times 15 \mathrm{~m}$, com uma distância mínima de 50 metros entre si, seguindo os critérios de uniformidade de topografia, densidade de indivíduos, cobertura de dossel e distância da floresta primária. Todas as árvores presentes nas parcelas, com diâmetro à altura do peito $(\mathrm{DAP}) \geq 5 \mathrm{~cm}$ foram medidas, identificadas e mapeadas em outubro de 1996. As plantas foram identificadas até espécie ou classificadas como morfoespécies e gêneros por falta de material fértil. Também houveram quatro morfoespécies nāo identificados. Para cada espécie foram calculados a área basal, freqüência, densidade absoluta e relativa, além do índice de valor de importância (IVI), segundo as equações de Krebs (1972). 


\section{Germinações no viveiro}

Em cada uma das 8 parcelas, foram retiradas 20 amostras aleatórias de solo ao final de outubro de 1996. Cada amostra de solo consistia de um quadrado de $20 \times 20 \mathrm{~cm}$ a uma profundidade de $3 \mathrm{~cm}$. As amostras foram lavadas em água corrente e passadas por peneiras de solo com aberturas de $2 \mathrm{~mm}, 1 \mathrm{~mm}$ e $500 \mathrm{~mm}$, segundo o método de Heerdt et al. (1996). Nesse método apenas galhos, folhas e pedras são retirados. Quando havia frutos inteiros, eles eram triturados e reintegrados às peneiras. Para servir de testemunho de que as sementes muito pequenas nāo passariam pela peneira de $500 \mathrm{~mm}, 5$ amostras foram lavadas e passadas em peneiras de $250 \mathrm{~mm}$ e foram postas em bandejas para se observar a germinação. Nenhuma semente germinou, e portanto consideramos que peneiras de $500 \mathrm{~mm}$ eram suficientes para reter todas as sementes. Além disso, outras 5 amostras extras foram escolhidas aleatoriamente e observadas na lupa após lavagem em peneiras de $250 \mathrm{~mm}$ e nenhuma semente foi vista.

Após a lavagem as amostras foram transferidas para bandejas plásticas, contendo solo previamente esterilizado em auto-clave por 3 horas. O resíduo das peneiras foi depositado sobre o solo das bandejas em novembro de 1996. Alguns locais na bandeja foram usados como controle, sem nada colocado para se estimar a contaminação externa. As bandejas foram levadas à casa de germinação para acompanhar a emergência de plântulas. As bandejas foram regadas em dias alternados até a saturaçāo do solo e as germinaçōes acompanhadas a cada 15 dias. Após a contagem alguns indivíduos dos morfotipos não identificados foram transplantados para copos plásticos individuais para posterior identificação. O acompanhamento se estendeu por um período de 3 meses, de janeiro à abril de 1997. A identificação das plântulas foi feita com o auxílio de espécimens coletados no campo e por comparação com exsicatas presentes na coleção do laboratório da ORSTOM - INPA, além de investigaçāo junto à Coordenação de Pesquisas em Ciências Agronômicas do INPA.

\section{Germinações no campo}

Ao final de outubro de 1996, todas as árvores com $\operatorname{DAP}^{3} 5 \mathrm{~cm}$ foram cortadas com moto-serra e removidas das oito parcelas para estimular germinações do banco de sementes no campo. Foram estabelecidas 3 sub-parcelas de $0,50 \times 0,50 \mathrm{~m}$ em cada parcela, as quais foram cobertas com tecido transparente a altura de 1.5 $\mathrm{m}$, que permitia a entrada de luz e água mas impedia a entrada de sementes via chuva de sementes. Após o corte da vegetação, os indivíduos presentes nas sub-parcelas provenientes do banco de sementes foram monitorados a 41, 83, 108 e 138 dias, pois durante este período todos os indivíduos emergentes foram individualizados por marcações com etiquetas plásticas numeradas.

\section{RESULTADOS}

\section{Composição da floresta secundária}

Do total de 458 árvores registradas com DAP $^{3} 5 \mathrm{~cm}$ nas 8 parcelas, $79,0 \%(\mathrm{n}=362)$ eram Vismia (V cayennensis, V guianensise $V$. japurensis) (Tabela 1). Mais 17 espécies ocorreram nas parcelas, sendo as mais comuns: Goupia glabra (Celastraceae), Laetia procera (Flacourtiaceae) e Bellucia sp. (Melastomataceae). 
Tabela 1. Parâmetros fitossociológicos das espécies amostradas em uma capoeira de 8 anos, dominada por Vismia sp., ordenados segundo o valor de importância: FR-frequência relativa (\%), DR-densidade relativa (\%), DoR-dominância relativa (\% area basal) e IVI-índice de valor de importância, de 458 indivíduos e $36.609 \mathrm{~cm}^{2}$ de área basal.

\begin{tabular}{lcccc}
\hline Taxon & FR & DR & DoR & IVI \\
\hline Vismia cayannensis (Jacq.) Pers. & 13,11 & 40,82 & 37 & 90,9 \\
Vismia guianensis (Aubl.) Choisy & 13,11 & 24,23 & 30,53 & 67,8 \\
Vismia japurensis Reich. & 11,47 & 13,97 & 10,7 & 36,1 \\
Goupia glabra Aubl. & 13,11 & 10 & 8,4 & 31,5 \\
Laetia procera (Poepp.) Eichler & 11,47 & 4,36 & 5,6 & 21,44 \\
Bellucia spp. & 9,83 & 2,18 & 4 & 16 \\
Flacourtiaceae sp.1 & 3,27 & 0,65 & 0,41 & 4,34 \\
Miconia spp. & 3,27 & 0,43 & 0,38 & 4 \\
Casearia spp. & 3,27 & 0,65 & 0,36 & 3,9 \\
Cecropia sciadophylla Mart. & 1,63 & 0,22 & 0,93 & 2,7 \\
Inga sp. 1 & 1,63 & 0,43 & 0,63 & 2,7 \\
Stryphnodendrum sp. 1 & 1,63 & 0,22 & 0,64 & 2,5 \\
Lauraceae sp. 1 & 1,63 & 0,22 & 0,12 & 1,97 \\
Annonaceae sp. 2 & 1,63 & 0,22 & 0,11 & 1,97 \\
Não identificada 3 & 1,63 & 0,22 & 0,08 & 1,94 \\
Não identificada 1 & 1,63 & 0,22 & 0,07 & 1,92 \\
Guatteria sp. 1 & 1,63 & 0,22 & 0,07 & 1,92 \\
Não identificada 4 & 1,63 & 0,22 & 0,06 & 1,92 \\
Annonaceae sp. 1 & 1,63 & 0,22 & 0,05 & 1,91 \\
Rubiaceae sp.1 & 1,63 & 0,22 & 0,05 & 1,91 \\
$\quad$ Total Relativo & 100 & 100 & 100 & 300 \\
\hline
\end{tabular}

Juntas, as 3 espécies de Vismia somaram $77,3 \%$ da área basal total, seguidas de $G$. glabra e L. procera (Tabela 1). As três espécies de Vismia foram as mais importantes, sendo V. cayennensis a mais importante com 30,3 $\%$, seguida de $V$. guianensis com $22,6 \%$ e $V$. japurensis com $12,0 \%$. Vismia japurensis foi a única espécie do gênero que não ocorreu em todas as parcelas, estando ausente em uma delas. Logo abaixo em importância, vêm as espécies G. glabra com $31,5 \%$, L. procera com $21,4 \%$, e Bellucia sp. com 16,0\%.
Das outras 14 espécies amostradas, sete estavam representadas por apenas 1 indivíduo, e quatro por 2 a 3 indivíduos, com IVI variando de 1-4\%, freqüência relativa entre $1-4 \%$, densidade relativa entre $0,2-0,65 \%$ e dominância relativa entre $0,05-0,9 \%$ (Tabela 1 ).

As 3 espécies de Vismia apresentaram maior número de indivíduos em quase todas as classes de diâmetro em relação aos demais gêneros, sendo que a maior diferença esteve entre as classes intermediárias de $9-14 \mathrm{~cm}$ de diâmetro. Assim a distribuição dos outros 
gêneros arbóreos seguiu a padrão clássico de “ J” invertido, enquanto Vismia apresentou uma distribuição mais homogênea dos indivíduos na maioria das classes de DAP (Fig. 1). As duas distribuções não mostraram diferença estatística significante (Kolmogorov-Smirnov teste, $\mathrm{p}=$ $0,30)$.

\section{Germinações no viveiro}

Do total de 160 amostras de solo coletadas, apenas 32 foram usadas na descrição do banco de sementes do campo e 48 foram usadas para descrever as áreas de coleta. As demais amostras foram desconsideradas em decorrência da morte das plântulas presentes por falta de regas durante uma semana. O total de 10.350 sementes foi dividido por área superficial $\left(32 \times 0,04 \mathrm{~m}^{2}\right)$, resultando numa média de 8.085 sementes $/ \mathrm{m}^{2}$. Das 17 espécies e morfotipos presentes, metade foi composto por ervas e cipós, e outra metade por espécies arbóreas e arbustivas (Tabela 2). Entre as arbóreas as mais comuns em ordem de densidade, foram as três espécies de Vismia (Clusiaceae), Bellucia (Melastomataceae), várias espécies de Cecropia (Cecropiaceae) e Trema micrantha (Ulmaceae), e entre as arbustivas foram: Piper (Piperaceae), Solanum rugosum (Solanaceae) e $S$. crinitum (Solanaceae). Entre as ervas as espécies mais abundantes foram Gramineae, Borreria latifolia (Rubiaceae), B. verticilata e Croton trinitatus (Euphorbiaceae). Ao todo ocorreram 10 taxa arbóreos-arbustivos, 5 ervas e 2 cipós.

\section{Germinações no campo}

Um total de 2.612 indivíduos germinados sobreviveram até 138 dias em 24 réplicas de $0,25 \mathrm{~m}^{2}$ acompanhadas no campo, correspondendo a 435 sementes $/ \mathrm{m}^{2}$, muito menos germinaçōes do que as 8,085 sementes/ $\mathrm{m}^{2}$ observadas no viveiro (teste-t, $\mathrm{P}=0.001$, $\mathrm{df}=54$ ). Houve uma grande similaridade na composição específica entre os dois tipos de amostras, mantidas no campo e em casa de vegetaçāo. Das 16 taxa identificados nas subparcelas do campo apenas três espécies não germinarem no viveiro. Das 17 presentes no viveiro, quatro nāo germinaram no campo (Tabela 2). A similaridade nas germinações de campo e de viveiro foi confirmada pelo índice de Sorenson qualitativo $(0,79)$ e de Sorenson quantitativo $(0,63)$ usando abundâncias relativas. Vismia teve o maior número de indivíduos das germinações do campo. Já as gramíneas, seguidas por Piper sp. dominaram o banco de sementes acompanhado em viveiro (Tabela 2). Porém a forma de vida das plantas germinadas foi muito similar entre o campo e o viveiro (Tabela 2).

O tempo de sobrevivência não foi regular ao longo do período observado no campo. De um total de 336 plantas acompanhadas e individualizadas à 41 dias após o corte da vegetação, sobreviveram $83,3 \%$ à 83 dias, caindo para $63,0 \%$ à 108 dias e se mantendo até 138 dias.

Nāo houve nenhum representante de espécie de mata primária em todas as parcelas amostradas, tanto no solo coletado como nas sub-parcelas cobertas acompanhadas diretamente no campo. Apenas $20 \%$ das espécies arbóreas $(n=20)$ identificadas na vegetação original esteve presente no banco de sementes.

\section{DISCUSSÃO}

A área de estudo não apresentou grande heterogeneidade na estrutura florestal nem em 
Tabela 2. Número de indivíduos germinando do banco de sementes no campo e do solo colocado no viveiro, durante 138 dias de observação.

\begin{tabular}{llll}
\hline Táxon & Do Campo & Do Viveiro & Forma de Vida \\
\hline Apocynaceae sp. 1 & 0 & 100 & cipó \\
Bellucia spp. & 92 & 550 & árvore \\
Borreria spp. & 0 & 225 & erva \\
Borreria latifolia (Aubl.) K. Schum & 192 & 175 & erva \\
Borreria verticillata (L.) G.F.W. Meyer & 28 & 400 & erva \\
Cecropia spp. & 28 & 425 & árvore \\
Cecropia purpurascens C.C. Berg & 4 & 0 & árvore \\
Croton trinitatus Mill. & 24 & 0 & arbusto \\
Cipós & 36 & 100 & cipo \\
Gramineae spp. & 324 & 2150 & erva \\
Palicourea sp. & 4 & 0 & árvore \\
Pteridium aquilinum (L.) Kuhn & 4 & 75 & erva \\
Phyllantus sp. 1 & 0 & 350 & árvore \\
Piper sp. 1 & 72 & 2025 & arbusto \\
Solanum spp. & 0 & 200 & arbusto \\
Solanum critinum Lam & 8 & 25 & árvore \\
Solanum rugosum Dunal & 16 & 575 & arbusto \\
Stachytarphetta cayennensis (L.C. Rich.) Vahl & 8 & 725 & arbusto \\
Trema micrantha (L.) Blume & 4 & 150 & árvore \\
Vismia spp. & 1604 & 375 & árvore \\
Taxa não identificados & 164 & 1725 & - \\
Total de individuos & 2612 & 10350 & \\
Total de espécies & 16 & 17 & \\
& & & \\
\hline
\end{tabular}

espécies relacionadas à floresta primária (Mesquita et al., 2001). A grande maioria das árvores maiores que $5 \mathrm{~cm}$ de diâmetro pertenciam a um único gênero Vismia. A estratificação arbórea quase não existiu, aparentemente com poucas plantas regenerando no sub-bosque abaixo do dossel de Vismia. Além de Vismia spp. há poucas espécies arbóreas e palmeiras, sendo a área dominada exclusivamente por espécies dependentes de luz. Oliveira \& Mori (1999) encontraram 280 espécies arbóreas em 1 ha de floresta madura próximo à região deste estudo enquanto 1 ha de capoeira de Vismia sp. apresentou apenas 20 espécies.

A dominância das espécies de Vismia pode ser uma das causas do baixo número de espécies regenerando sob o seu dossel. Aparentemente, o número de indivíduos e espécies presentes no sub-bosque de uma capoeira dominada por Cecropia é maior do que o registrado para capoeiras de idade similar dominadas por Vismia (Williamson et al., 1998; Mesquita et al., 2001). As três espécies de Vismia têm a capacidade de rebrotar de raízes e conseqüentemente sobreviveram nas pastagens queimadas. Assim, são as primeiras árvores a formar o dossel na pastagem abandonada. Em contraste, a mata secundária seguida de desmatamento sem incêndio desenvolve um dossel misto com mais espécies de Cecropia do que de Vismia.

A dominância de poucas espécies pioneiras em florestas secundárias tropicais após queimadas vem sendo abordada por vários autores. Saldarriaga et al. (1988) encontraram em áreas de capoeiras de 9-14 anos da Colômbia e Venezuela uma dominância das 
espécies pioneiras Cecropia ficifolia, $V$. japurensis e V. lauriformis. Uhl et al. (1988) encontraram para uma capoeira de 8 anos derivada de pasto de uso leve, em Paragominas, Pará, Brasil, dominância de Cecropia sp. e Cordia bicolor, que somaram $43,2 \%$ do IVI total. Os mesmos autores encontraram para capoeira de idade similar, de uso moderado, a dominância de $V$ guianensis, Cecropia sp., $L$. procera e Solanum rugosum, somando quase $40 \%$ do IVI total.

Na floresta estudada, $V$ cayennensis, $V$. guianensis e $V$. japurensis são as espécies pioneiras mais importantes na determinação da homogeneidade do componente dominante. As espécies analisadas apresentam, sem exceção, características de pioneiras e em sua maioria pertencem a estágios iniciais da sucessão. A comunidade está em fase inicial de regeneração, por apresentar maior dominância (número de indivíduos) de espécies pioneiras, principalmente do gênero Vismia em todas as áreas amostradas. O baixo número de plântulas sob seu dossel pode indicar o caráter sucessional descrito por Hartshorn (1978), ou ser apenas estratégia de ocupação de interferência, que impediria o estabelecimento de plântulas de outras espécies (Williamson et al., 1998; Mesquita et al., 2001).

Das 17 espécies presentes nas parcelas estudadas só 5 foram de taxa arbóreos. O tempo médio de germinação foi entre 2 a 4 semanas, similar a outros estudos de banco de sementes (Cheke et al.;1979; Enright, 1985). A composição do banco de sementes refletiu a composiçāo original da área de estudo, que é marcada por espécies de estágios sucessionais, com dominância de Vismia spp. A história de uso da área deriva de pasto, com queimas sucessivas, e plantio de gramíneas para pastagem, a qual influenciou a composição do banco de sementes, além da entrada de sementes de espécies invasoras, como Borreria spp. e outras herbáceas. O uso poderia também limitar espécies arbóreas presentes na vegetação primária, como Laetia procera e Goupia glabra, que nāo estiveram presentes no banco de sementes coletado.

A ausência de espécies de mata primária na área pode estar relacionada à fatores como a distância da fonte primária, ecologia de dispersão das espécies, falta de recursos (abrigo, alimento) para dispersores, falta de corredores interligando a área de vegetação secundária à mata, falta de nutrientes no solo, ou ainda competição entre as espécies pioneiras e as de mata, favorecendo as pioneiras. Fatores como predaçāo e remoçāo de sementes, também impedem que sementes arbóreas de mata primária se estabeleçam na capoeira (Nepstad et al., 1990, 1996; Miriti, 1998).

Espécies pioneiras dominam o banco e a chuva de sementes em regiões tropicais e subtropicais (Garwood, 1989). Áreas de vegetação secundária têm mais sementes no solo que áreas de mata e a grande maioria são de pioneiras, pois essas espécies produzem sementes de fácil dispersāo e que se mantêm viáveis no solo por muito tempo (Cheke et al., 1979; Holthuijzen \& Boerboom, 1982; Enright, 1985). Gorchov et al. (1993) encontraram maior deposição de sementes de espécies pioneiras dispersadas por aves e morcegos, como Vismia sp. e Cecropia sp., em faixas de corte seletivo (strip-cutting) na Amazônia Peruana do que nas áreas adjacentes à mata primária. Fatores como facilidade de dispersão a longas distâncias, copiosa produção de sementes disponíveis a aves e morcegos, além de mecanismos de dormência pós-dispersão 
facilitam a permanência dessas espécies no banco de sementes em áreas de distúrbio. A maior disponibilidade de luz em ambientes abertos é outro fator essencial à germinação de pioneiras, que logo germinam e produzem sementes localmente incrementando o banco de sementes.

Em geral, espécies herbáceas e arbustivas são mais comuns em áreas alteradas e vegetaçāo secundária. Garwood (1989) descreveu uma grande proporção de espécies herbáceas no banco de sementes de áreas perturbadas, em várias regiões tropicais, como México, Costa Rica e Venezuela, todos variando entre 25 a $90 \%$ do total de espécies registradas. Cerca de $36 \%$ das espécies encontradas no banco de sementes da capoeira estudada eram herbáceas. Rico-Gray \& GarciaFranco (1992), estudando diversos estágios de regeneração (entre 1-20 anos, contando entre 8-67 espécies), em Yucatán, México, encontraram apenas 3 espécies arbóreas no banco de sementes, em todos os sítios amostrados. Nepstad et al. (1996) também encontraram um baixo número de espécies arbóreas presentes no banco de sementes em áreas de pastos abandonados no Pará, contribuindo com apenas $2 \%$ do total das germinaçōes. Os mesmos autores encontraram espécies em comum com este estudo, como Vismia sp., Cecropia sp., Solanum crinitum e Stachytarphettasp.

Porque as amostras do banco de sementes não tiveram espécies arbóreas da mata primária? A distância entre a capoeira estudada e a mata primária, aproximadamente $550 \mathrm{~m}$, poderia explicar a falta dessas espécies, já que a grande maioria delas são dispersas por animais que normalmente não circulam entre áreas abertas. Outro fator de impedimento à entrada dessas espécies nessas áreas é a intensa remoção de sementes e a predaçāo, além da competiçāo com gramíneas e espécies adaptadas a condições adversas, como falta de luz, água e nutrientes (Nepstad et al., 1996; Miriti, 1998).

$\mathrm{O}$ banco de sementes no viveiro mostrou quase vinte vezes mais germinaçōes por área do que em sub-parcelas cobertas. Isso pode ter ocorrido devido ao volume de solo coletado por área $(20 \times 20 \times 3 \mathrm{~cm})$ em cada parcela, que, ao ser revolvido e lavado, fez com que mais sementes germinem do que o solo acompanhado diretamente nas parcelas $(0,25$ $\mathrm{m}^{2}$ ). A coleta de solo foi feita à profundidade de $3 \mathrm{~cm}$, pois vários trabalhos têm descrito esta camada como de maior densidade de sementes viáveis $(0-5 \mathrm{~cm})$ (Cheke, 1979; Enright, 1985; Garwood, 1989; Leal, 1992; Mcgee \& Feller, 1993; Dalling et al. 1994). No entanto, é provável que sementes de pioneiras enterradas, mesmo a $3 \mathrm{~cm}$ de profundidade, não germinem tão facilmente. Neste caso, apenas aquelas depositadas nas camadas mais superficiais do solo teriam germinado nas sub-parcelas do campo. Isso explicaria pelo menos em parte a diferença no número de germinações entre viveiro e campo.

O período observado pode não ter sido extenso o suficiente para acompanhar todas as germinações do banco de sementes, mas pode dar um indicativo da sobrevivência das plantas estabelecidas oriundas de banco de sementes (Garwood, 1989). Além disso os intervalos entre visitas foram muito irregulares e longos, não permitindo verificar se ocorreu germinação e mortalidade de outros indivíduos entre esses intervalos. Isso em parte explicaria a grande diferença observada entre germinações no viveiro e no campo, já que os intervalos de visitas para o viveiro foram semanais, tendo assim mais registros de germinações no tempo 
que no caso do campo. Mas, no campo não houve uma mortalidade acentuada das plântulas nos intervalos observados, sendo que a taxa de sobrevivência permaneceu constante nos dois últimos períodos de observação, a 108 e a 138 dias.

Como conclusāo a composiçāo do banco de sementes estudado na área dominada por Vismia foi característica de estágios sucessionais, com forte influência do histórico de pastagem e que determinou a presença de espécies pioneiras, e um grande número de herbáceas. O gênero dominante da cobertura vegetal original, Vismia spp., também foi o dominante no banco de sementes observado diretamente no campo, mas não no viveiro. Desta forma, a regeneração da floresta primária deverá ser gradual e dependente de fontes próximas de sementes, além da presença de dispersores, que incrementem a chuva de sementes local. O banco de sementes refletiu somente a composição da vegetação estabelecida na área, sem conter espécies novas que pertençam exclusivamente ao banco de sementes.

\section{AGRADECIMENTOS}

A pesquisa foi financiada pelo PDBFF. Os pesquisadores receberam bolsas de mestrado (LMM) e de recém-doutor (RM) do Conselho Nacional de Desenvolvimento Científico e Tecnológico (CNPq), e bolsa de professor visitante (GBW) da CAPES e da Comissão Fulbright. Agradecemos aos técnicos de campo, Srs. João de Deus Fragata e Antonio Cardoso. Este trabalho é o número 382 na série técnica do PDBFF.

\section{BIBLIOGRAFIA CITADA}

Cheke, A.S.; Nanakorn, W.; Yankoses, C. 1979. Dormancy and dispersal of seeds of secondary forest species under the canopy of primary tropical rain forest in northern Thailand. Biotropica, 11(2):88-95.

Dalling, J.W.; Swaine, M.D.; Garwood, N.C. 1994. Effect of soil depth on seedling emergence in tropical soil seed-bank investigations. Functional Ecology, 9:119121.

Enright, N. 1985. Existence of a soil seed bank under rainforest in New Guinea. Australian Journal of Ecology, 10:67-71.

Garwood, N.C. 1989. Tropical soil seed banks: a review. In: Leck, M.A.; Parker, V.T.; Simpson, R.L. (Eds). Ecology of Soil Seed Banks. Academic Press, San Diego. p.149209.

Gorchov, D.L.; Cornejo, F.; Ascorra, C.; Jaramillo, M. 1993. The role of seed dispersal in the natural regeneration of rain forest after strip-cutting in Peruvian Amazon. Vegetatio, 107/108:339-349.

Hartshorn, G.S. 1978. Tree falls and the tropical forest dynamics. In: Tomlinson, P.B.; Zimmermann, M.H. (Eds). Tropical Trees as Living Systems. Cambridge University Press, NY. p.617-638.

Heerdt, G.N.J.; Verweij, G.L.; Bekker, R.M.; Bakker, J.P. 1996. An improved method for seed-bank analysis: seedling emergence after removing soil by sieving. Functional Ecology, 10:144-151.

Holthuijzen, A.M.A.; Boerboom, J.H.A. 1982. The Cecropia seedbank in the lowland rain forest. Biotropica, 14(1):62-68.

Krebs, C.J. 1972. The experimental analysis of distribution and abundance. Harper \& Row, New York. 396 p.

Leal Filho, N. 1992. Caracterização do banco 
de sementes de três estádios de uma sucessão vegetal na zona da mata de Minas Gerais. Dissertação de Mestrado. Universidade Federal de Minas Gerais, Viçosa, Minas Gerais. 116 p.

Lovejoy, T.E.; Bieregaard Jr., R.O. 1990. Central Amazonian forests and the minimum critical size of ecosystems project. In: Gentry, H.A. (Ed.) Four neotropical rainforests. Yale University Press, New Haven. p. 60-71.

Mesquita, R.C.M.; Ickes, K.; Ganade, G.; Williamson, G.B. 2001. Alternative successional pathways following deforestation in the Amazon Basin. Journal of Ecology, 89: 528-537.

McGee, A.; Feller, M.C. 1993. Seed banks of forested and disturbed soils in southwestern British Columbia. Canadian Journal of Botany, 71:1574-1583.

Miriti, M.A. 1998. Regeneração florestal em pastagens abandonadas na Amazônia central: competiçāo, predaçāo, e dispersāo de sementes. In: Gascon, C.; Moutinho, P. (Eds). Floresta Amazônica: Dinâmica, Regeneração e Manejo. Instituto Nacional de Pesquisas da Amazônia, Manaus, Amazonas. p. 179-190.

Nepstad, D.C.; Uhl, C.; Pereira, C.A.; Da Silva, J.M.C. 1996. A comparative study of tree establishment in abandoned pasture and mature forest of eastern Amazonia. Oikos, $76: 25-39$.

Nepstad, D.C.; Uhl, C.; Serrāo, E.A. 1990. Surmounting barriers to forest regeneration in abandoned highly degraded pasture: a case study from Paragominas, Pará, Brazil. In: Anderson, A.B. (Ed). Alternatives to
Deforestation: Steps toward Sustainable Use of the Amazon Rain Forest. Columbia University Press, NY. p. 215-229.

Oliveira, A.A.; Mori, S.A. 1999. A central Amazonian terra firme forest. I. High tree species richness on poor soils. Biodiversity and Conservation, 8:1219-1244.

Rankin-de-Merona, J.M.; Prance, G.T.; Hutchings, R.W.; Da Silva, M.F.; Rodrigues, W.A.; Veühling, M.E. 1992. Preliminary tree inventory results from upland rain forest of the Central Amazon. Acta Amazonica, 22: 493-534.

Rico-Gray, V.; García-Franco, J.G. 1992. Vegetation and soil seed bank of successional stages in tropical lowland deciduous forest. Journal of Vegetation Sima?

617-624.

Rodrigues, F.C.M.; Costa, L.G.S.; Reis, A. 1990. Estratégias de estabelecimento de espécies arbóreas e o manejo de florestas tropicais. In: Anais do $6^{\circ}$ Congresso Florestal Brasileiro, Campos do Jordão. Sociedade Brasileira de Silvicultura (SBS) e Sociedade Brasileira de Engenheiros Florestais (SBEF), Campos do Jordão, p. 676-684.

Saldarriaga, J.G.; West, D.C.; Tharp, M.L.; Uhl, C. 1988. Long term chronosequence of forest succession in the upper Rio Negro of Colombia and Venezuela. Journal of Ecology, 77:938-958.

Santos, H.M. 1968. Balanço hídrico de Manaus, Amazonas. Conselho Nacional de Pesquisas, Instituto Nacional de Pesquisas da Amazônia, Manaus, Amazonas, Brasil. 
Thompson, K. 1979. Occurrence of viable seeds in relation to environmental gradients. Journal of Biogeography, 5:425-430.

Thompson, K. 1992. The functional ecology of seed banks. In: Fenner, M. (Ed). Seeds: the Ecology of Regeneration in Plant Communities. CAB Internacional, Wallingford. p. 231-258.

Uhl, C.; Clark, K. 1983. Seed ecology of selected Amazon Basin successional species. Botanical Gazette, 144: 419-425.

Uhl, C.; Buschbacher, R.; Serrão, E.A.S. 1988. Abandoned pastures in Eastern Amazonia. I. Patterns of plant succession. Journal of Ecology, 76: 663-681.

Vázquez-Yanes, C. 1980. Light quality and seed germination in Cecropia obtusifolia and Piper auritum from a tropical rain forest in Mexico. Phyton, 38(1):33-35.
Viera, I.C. 1996. Forest succession after shifting cultivation in Eastern Amazonia. Tese de Doutorado, University of Stirling, Scotland. 205 p.

Williams-Linera, G. 1993. Soil seed banks in four lower montane forests of Mexico. Journal of Tropical Ecology, 9:321-337.

Williamson, G.B.; Mesquita, R.C.G.; Kalan, I.; Ganade, G. 1998. Estratégias de árvores pioneiras nos Neotrópicos. In: Gascon, C.; Moutinho, P. (Eds). Floresta Amazônica: Dinâmica, Regeneração e Manejo. Instituto Nacional de Pesquisas da Amazônia, Manaus, Amazonas. p. 131144.

Submetido à publicação: 16/08/1999.

Aceito: 25/10/2002. 